\title{
A practical analytical solution for one-dimensional consolidation
}

\author{
M. M. STICKLE and M. PASTOR
}

\begin{abstract}
In this work a four-step strategy to derive an analytical solution to the one-dimensional consolidation equation under a general time-dependent loading is presented. The strategy is based on the eigenfunction expansion method. Most existing solutions in the specialised literature are developed for a particular type of loading, whereas the proposed strategy can be easily applied to obtain an accurate response for a general loading. In order to assess the validity of the proposed strategy, it is applied to constant, single ramp, cyclic square and cyclic haversine loading profiles. The results are in agreement with the analytical solution previously obtained by other researchers.
\end{abstract}

KEYWORDS: consolidation; theoretical analysis

\section{INTRODUCTION}

Consolidation is a gradual process which involves, simultaneously, drainage, compression and stress transfer (Taylor, 1948). In other words, consolidation is a quasi-static transient process in which there is coupling between the fluid flow and the soil skeleton while effective stresses are updated (Wood, 2004). A key aspect in order to develop accurate models, which are capable of reproducing the principal features of this phenomenon, is the adequate description of multiphase porous media. Such multiphase materials can be properly described within the thermodynamically consistent theory of porous media (TPM), where all constituents are assumed to occupy the space simultaneously (de Boer, 2005).

Exact solutions of the coupled partial differential equations that arise from TPM not only illustrate the response of saturated porous media, but also can be used to validate the accuracy of various numerical results

A theory to explain the one-dimensional consolidation process, where the pore fluid flow as well as the skeleton deformation are constrained to take place in the vertical direction, was first proposed by Terzaghi (1925). Based on Terzaghi's work, Biot (1941) proposed a close analytical solution for the one-dimensional consolidation process in a finite length column of soil under constant loading.

In practice, external surface loads may be time dependent. To analyse time-dependent loading, a graphical construction method was suggested by Terzaghi (1943). Olson (1977) presented charts for one-dimensional consolidation for the case of simple ramp loading. Zienkiewicz and coworkers (Zienkiewicz et al., 1980) presented a set of solutions for the transient response under periodic loading. Other researchers (Baligh \& Levadoux, 1978; Favaretti \& Soranzo, 1995; Guan et al., 2003; Geng et al., 2006) derived solutions for different types of cyclic loading including rectangular, trapezoidal, triangular and sinusoidal cyclic loading. Razouki et al. (2013) proposed an analytical solution for one-dimensional consolidation under haversine cyclic loading.
All the above-mentioned solutions were intended for a particular type of loading. As mentioned in Razouki \& Schanz (2011) and Razouki et al. (2013) exact analytical solutions for a complex loading profile are not easy to achieve. To overcome this drawback, Conte \& Troncone (2006) proposed the expansion of a periodic time-dependent loading as a classical Fourier series, computing the pore pressure distribution for each harmonic component by way of Duhamel's principle and then superposing all the calculated terms. As will be clarified later in this paper, in the authors' opinion the four-step solution strategy presented in this work is easier than and (at least) of the same order of accuracy as the one derived by Conte and Troncone in 2006.

In the present work the authors propose a four-step strategy, based on the eigenfunction expansion method, to derive an analytical solution to the one-dimensional consolidation equation under a general time-dependent loading. The solution provided allows easy implementation of semianalytical expressions that can be applied to general loading profiles that might appear in practical cases. To the best of the authors' knowledge, this solution strategy has not been previously proposed for one-dimensional consolidation.

The paper is structured as follows. For the sake of completeness, in the next section the consolidation equations of a fluid saturated porous medium within the TPM (de Boer et al., 1993; de Boer, 2005; Stickle et al., 2016) are summarised. In the third section, the analytical solution of the consolidation equation under a general time-dependent loading, by means of the eigenfunction expansion method, is fully derived. This section ends summarising the four steps to be followed to obtain the response under a general time-dependent loading. In the fourth section, the proposed strategy is applied to constant, single ramp, cyclic square and cyclic haversine loading profiles. Comparison with an analytical solution, as previously obtained by other researchers, is also presented in the fourth section. Some conclusions are established in the fifth and final section.

\section{CONSOLIDATION EQUATION FOR SATURATED SOILS}

The assumptions considered to obtain the full set of governing equations are listed below. (a) Porous medium is fully saturated.

(b) Solid and fluid phases are intrinsically incompressible.

(c) Neither mass nor heat exchanges between the solid and the fluid phase are considered. 
(d) Internal viscosity force within the fluid is neglected as compared with the viscous resistance opposed by the internal walls of the porous medium.

(e) Permeability is considered isotropic.

( $f$ ) Porous solid is considered isotropic and linear elastic under small strain regime.

(g) All dynamic terms, including convective terms, are neglected.

(h) Only loading by external forces is considered. Body forces are excluded.

Within the framework of TPM, a fluid saturated porous medium is described by two superimposed but immiscible phases. Each phase is composed by particles $P^{\alpha}(\alpha=f, s)$, where the superscript $\alpha$ denotes pore fluid for $\alpha=f$ and solid skeleton for $\alpha=s$.

The kinematics in TPM is based on the assumption (de Boer, 2005) that the spatial position $\boldsymbol{x}$ in the current configuration at time $t$ is simultaneously occupied by particles $P^{\alpha}$ of both constituents $(\alpha=f, s)$. Each particle $P^{\alpha}(\alpha=f, s)$ proceeds from its own reference position $\boldsymbol{X}^{\alpha}$ in the reference configuration at time $t_{0}$. Therefore, each phase has its own motion $\chi^{\alpha}$ and at the common spatial position $\boldsymbol{x}$ the phases interact (Fig. 1). In other words, the following relation holds at time $t$

$$
\boldsymbol{x}=\chi^{s}\left(\boldsymbol{X}^{s}, t\right)=\chi^{f}\left(\boldsymbol{X}^{f}, t\right)
$$

Within the modern TPM, the notion of superimposed phases along the control space relies upon the concept of volume fraction $n^{\alpha}(\boldsymbol{x}, t)$ of the phase $\alpha$ defined by

$$
n^{\alpha}(\boldsymbol{x}, t)=\frac{\mathrm{d} v^{\alpha}}{\mathrm{d} v}
$$

where $\mathrm{d} v^{\alpha}$ is the element volume of the phase $\alpha$ in the current configuration within a representative element volume $\mathrm{d} v$ of the overall porous media.

Within the framework of TPM, according to the abovementioned assumptions $(a)$ to $(h)$, the full set of coupled partial differential equations that governs the consolidation phenomenon for saturated soils is the following (de Boer et al., 1993; de Boer, 2005; Stickle et al., 2016)

$$
\text { Mixture momentum balance } \operatorname{div}(\boldsymbol{\sigma})=0
$$

Mixture volume balance $\operatorname{div}\left(\boldsymbol{v}^{s}+n^{f} \boldsymbol{v}^{f s}\right)=0$

together with the following equations to close the system

$$
\text { Darcy's law } \quad n^{f} \boldsymbol{v}^{f s}=-\frac{k^{f}}{\rho^{f R} \boldsymbol{g}} \operatorname{grad}\left(p^{f}\right)
$$

$$
\text { Terzaghi's principle } \quad \boldsymbol{\sigma}=\boldsymbol{\sigma}^{\prime}-p^{f} \boldsymbol{I}
$$

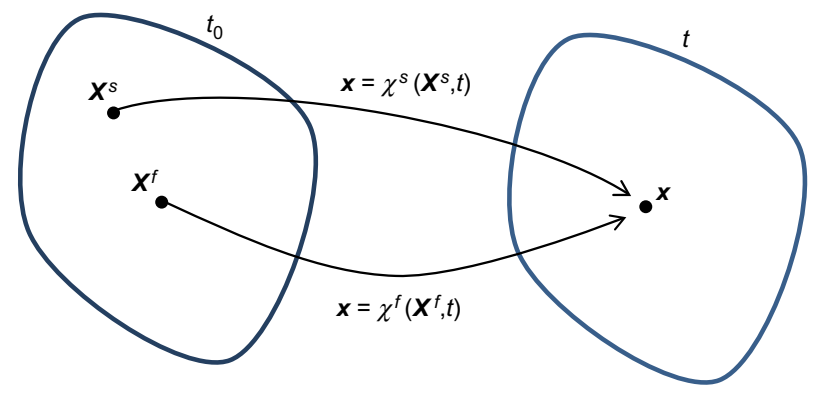

Fig. 1. Kinematical basic assumption within the TPM

$$
\begin{aligned}
& \text { solid constitutive relation } \quad \boldsymbol{\sigma}^{\prime}=\lambda(\boldsymbol{\varepsilon}: \boldsymbol{I}) \boldsymbol{I}+2 \mu \boldsymbol{\varepsilon} \\
& \text { solid strain tensor } \quad \boldsymbol{\varepsilon}=\frac{1}{2}\left[\operatorname{grad}\left(\boldsymbol{u}^{s}\right)+\operatorname{grad}^{\mathrm{T}}\left(\boldsymbol{u}^{s}\right)\right]
\end{aligned}
$$

where $\boldsymbol{\sigma}$ in equation (3) is the total Cauchy stress second-order tensor, while $\boldsymbol{v}^{s}$ in equation (4) is the velocity of the particles $P^{s}$ defined by

$$
\boldsymbol{v}^{s}=\frac{\partial \chi^{s}\left(\boldsymbol{X}^{s}, t\right)}{\partial t}
$$

The vector field $\boldsymbol{v}^{f s}=\boldsymbol{v}^{f}-\boldsymbol{v}^{s}$ in equations (4) and (5) is the relative velocity of the pore fluid to the solid skeleton, $k^{f}$ is the Darcy permeability, $\rho^{f R}$ is the intrinsic density of the pore fluid, $\boldsymbol{g}$ is the acceleration of gravity and $p^{f}$ is the pore fluid pressure. The symbol $\boldsymbol{\sigma}^{\prime}$ in equation (6) refers to the effective Cauchy stress second-order tensor, while $\lambda$ and $\mu$ in equation (7) are the macroscopic Lamé constants. The second-order tensor $\boldsymbol{\varepsilon}$ in equation (8) is the small strain tensor defined by means of the skeleton displacement vector $\boldsymbol{u}^{s}=\boldsymbol{x}-\boldsymbol{X}^{s}$.

The symbol grad ( $\square$ ) in equation (5) means the gradient operator with respect to the spatial position $\boldsymbol{x}$, while the operator div ( $\square$ ) in equations (3) and (4) is the divergence associated with grad $(\square)$.

The primary variables for this system are $\boldsymbol{u}^{s}$ and $p^{f}$. Appropriate initial and boundary conditions should be specified for this set of equations in order to have a correct physical meaning.

Concerning the boundary conditions, a fully saturated mixture is considered occupying the domain $\Omega$, where its boundary $\partial \Omega$ is decomposed in the following two different ways (Hughes, 2000)

$$
\partial \boldsymbol{\Omega}=\overline{\partial \boldsymbol{\Omega}_{\boldsymbol{u}^{s}} \cup \partial \boldsymbol{\Omega}_{\boldsymbol{t}}}=\overline{\partial \boldsymbol{\Omega}_{p^{f}} \cup \partial \boldsymbol{\Omega}_{q}}
$$

where the overline denotes closure while $\partial \Omega_{\boldsymbol{u}^{s}}$ and $\partial \Omega_{\boldsymbol{t}}$ are non-intersecting parts of the whole boundary $\partial \Omega$ on which solid displacement and total stresses are prescribed, respectively. On the other hand, $\partial \Omega_{p^{f}}$ and $\partial \Omega_{q}$ are non-intersecting parts of the whole boundary $\partial \Omega$ on which pore fluid pressure and fluid flux are prescribed, respectively. The following boundary conditions are prescribed in the above-mentioned parts of the boundary

$$
\text { Solid displacement specified } \boldsymbol{u}^{s}=\hat{\boldsymbol{u}}^{s} \text { on } \partial \boldsymbol{\Omega}_{\boldsymbol{u}^{s}}
$$

Total stress specified $\boldsymbol{\sigma} \boldsymbol{n}=\hat{\boldsymbol{t}}$ on $\partial \boldsymbol{\Omega}_{\boldsymbol{t}}$

Pore fluid pressure specified $p^{f}=\hat{p}^{f}$ on $\partial \Omega_{p^{f}}$

Fluid flux specified $\quad n^{f} \boldsymbol{v}^{f s} \cdot \boldsymbol{n}=\hat{q}$ on $\partial \Omega_{q}$

where $\hat{\boldsymbol{u}}$ and $\hat{\boldsymbol{t}}$ are given space and time vector functions, while $\hat{p}$ and $\hat{q}$ are given space and time scalar functions. The vector $\boldsymbol{n}$ is the outward unit normal vector to the boundary $\partial \Omega$ and $n^{f} v^{f s}$ is the Darcy velocity.

\section{ANALYTICAL SOLUTION IN FOUR STEPS}

The balance equations (3) and (4) are usually solved by using numerical methods. However, in the case of onedimensional small strain, an analytical solution is possible. In this section a procedure to obtain the analytical solution of the response of a one-dimensional column of length $L$, composed by a fluid-saturated poroelastic material and subjected to a general time-dependent loading $f(t)$, is derived. 
The description of the one-dimensional problem is as follows. The load is applied at the upper boundary of the column, which is perfectly drained. The motion of the solid skeleton within the column is constrained to take place along the vertical direction, defined by the $z$ axis, which is considered normal to the top boundary and pointing downwards (Fig. 2).

The bottom of the column is considered impermeable and fixed. Therefore, a standard PTIB (permeable top, impermeable bottom) column is considered. Zero vertical displacement is assumed through the column at initial time.

For a one-dimensional problem the governing equations (3)-(8) can be expressed as

$$
\begin{aligned}
& (\lambda+2 \mu) u_{z z}(z, t)-p_{z}(z, t)=0 \\
& u_{t z}(z, t)-\frac{k}{\rho \boldsymbol{g}} p_{z z}(z, t)=0
\end{aligned}
$$

In equations (13) and (14) the vertical value of $\boldsymbol{u}^{s}$ has been replaced by $u$, the fluid pressure $p^{f}$ by $p$, the Darcy's permeability $k^{f}$ by $k$ and the intrinsic density of the fluid phase $\rho^{f R}$ by $\rho$. Subscript $z$, as in $p_{z}$ or $u_{z z}$, stands for partial differentiation with respect to the spatial coordinate $z$, while the subscript $t$, for example in $u_{t z}$, denotes partial differentiation with respect to time. Appropriate initial and boundary conditions should be specified along with equations (13) and (14). The above description of the problem means that the following boundary and initial conditions are considered

$$
\begin{aligned}
& p \text { boundary conditions, } p(0, t)=0, p_{z}(L, t)=0 \\
& u \text { boundary conditions, } \sigma(0, t)=-f(t), u(L, t)=0 \\
& \text { initial conditions } u(z, 0)=0, p(z, 0)=f(0)
\end{aligned}
$$

It should be noted that extension of the present procedure to the case of a PTPB column, this is with a permeable top and bottom, would require only the modification of the condition of the second expression in equation (15) to be $p(L, t)=0$.

By integrating equation (13) with respect to $z$ the following relation between $u$ and $p$ can be obtained

$$
(\lambda+2 \mu) u_{z}(z, t)-p(z, t)=h(t)
$$

by equations (6), (7), the first expression in equation (15) and the first expression in equation (16), it can be seen

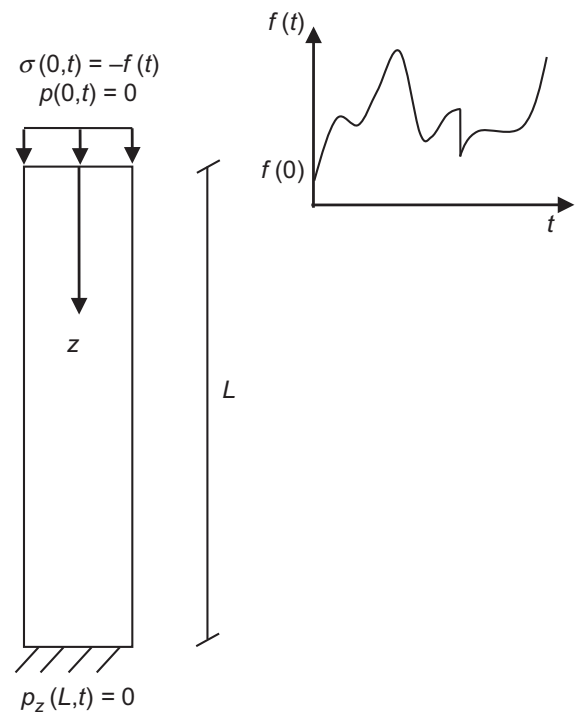

Fig. 2. Geometry and boundary conditions of the investigated problem that $h(t)$ is $-f(t)$. Therefore, equation (18) can be rewritten as

$$
(\lambda+2 \mu) u_{z}(z, t)-p(z, t)=-f(t)
$$

taking the partial derivative with respect to $t$ in equation (19) and bearing equation (14) in mind, the well-known diffusionlike equation governing the pore pressure distribution is obtained

$$
p_{t}(z, t)=c_{\mathrm{v}} p_{z z}(z, t)+f^{\prime}(t)
$$

where $c_{\mathrm{v}}=k(\lambda+2 \mu) / \rho g$ is the coefficient of consolidation in the vertical direction, while $\lambda+2 \mu$ is the oedometer modulus. In order to solve equation (20) the following initial and boundary conditions are considered

$$
\text { boundary conditions } \quad p(0, t)=0, p_{z}(L, t)=0
$$

$$
\text { initial condition } \quad p(z, 0)=f(0)
$$

The initial boundary value problem defined by equations (20)-(22) has homogeneous boundary conditions. However the partial differential equation (20) is nonhomogeneous owing to the presence of $f^{\prime}(t)$. In order to solve this problem, first the corresponding homogeneous problem

$$
\begin{aligned}
& p_{t}(z, t)=c_{\mathrm{v}} p_{z z}(z, t) \\
& p(0, t)=0, \quad p_{z}(L, t)=0
\end{aligned}
$$

should be addressed. What is required from this homogeneous problem are the eigenvalues and eigenfunctions of the related Sturm-Liouville problem when separation of variables is considered (Taylor, 1948; Farlow, 1982; Selvadurai, 2000; Logan, 2004). In the present case the following eigenvalues and eigenfunctions are obtained, respectively

$$
\begin{aligned}
& \lambda_{n}=\left[\frac{(1+2 n) \pi}{2 L}\right]^{2} \text { for } n=0,1, \ldots \\
& y_{n}=\sin \left[\frac{(1+2 n) \pi z}{2 L}\right] \text { for } n=0,1, \ldots
\end{aligned}
$$

Once the eigenvalues and eigenfunctions of the associated homogeneous problem are known, the solution $p(z, t)$ along with the time derivative of the external loading $f^{\prime}(t)$ are expressed as a linear combination of the eigenfunctions $y_{n}$, yielding

$$
\begin{aligned}
& p(z, t)=\sum_{n=0}^{\infty} p_{n}(t) \sin \left[\frac{(1+2 n) \pi z}{2 L}\right] \\
& f^{\prime}(t)=\sum_{n=0}^{\infty} f_{n}(t) \sin \left[\frac{(1+2 n) \pi z}{2 L}\right]
\end{aligned}
$$

where the coefficients $p_{n}(t)$ are to be determined while $f_{n}(t)$ are the Fourier coefficients of $f^{\prime}(t)$ with respect to the orthogonal family of eigenfunctions, equation (26), and can be computed in the present case as

$$
f_{n}(t)=\frac{4 f^{\prime}(t)}{\pi(2 n+1)}
$$

By substituting equations (27) and (28) into equations (20)-(22) the following initial value problem is obtained for the coefficients $p_{n}(t)$

$$
p_{n}^{\prime}(t)+\aleph_{n} p_{n}(t)=f_{n}(t)
$$




$$
p_{n}(0)=\frac{4 f(0)}{\pi(2 n+1)}
$$

where $\aleph_{n}=c_{\mathrm{v}}[(1+2 n) \pi / 2 L]^{2}$ while $f_{n}(t)$ are given by equation (29). By integrating factor $\mathrm{e}^{\aleph_{n} t}$, the following solution of the ordinary differential equation (30) might be obtained

$$
p_{n}(t)=\frac{4}{\pi(2 n+1)}\left[\mathrm{e}^{-\aleph_{n} t} f(0)+\int_{0}^{t} \mathrm{e}^{-\aleph_{n}(t-\tau)} f^{\prime}(\tau) \mathrm{d} \tau\right]
$$

where equation (29) has been already considered.

Finally, the fluid pressure distribution along the column will be obtained once the integral within equation (32) is computed. In the case where the loading profile is smooth, direct application of equation (32) can be performed. However, if the loading profile is a piecewise smooth function, that is, a function not necessarily smooth but its graph is made up of finitely many smooth pieces, with $\left\{t_{i}, i=1, \ldots, m\right\}$ points of simple jump discontinuity (Logan, 2004), it is possible to consider the following representation

$$
\begin{aligned}
f(t)= & f_{1}(t)\left[1-H\left(t-t_{1}\right)\right]+f_{m+1}(t) H\left(t-t_{m}\right)+\cdots \\
& \sum_{i=1}^{m-1} f_{i+1}(t)\left[H\left(t-t_{i}\right)-H\left(t-t_{i+1}\right)\right]
\end{aligned}
$$

where $f_{i+1}(t)$ are continuous functions on $\left[t_{i}, t_{i+1}\right]$ and have continuous first derivative on $\left(t_{i}, t_{i+1}\right)$ such that $f(t)=f_{i+1}(t)$ for $t \in\left(t_{i}, t_{i+1}\right)$, while the Heaviside function $H$ is defined by

$$
H(t)= \begin{cases}1 & \text { if } t \geq 0 \\ 0 & \text { if } t<0\end{cases}
$$

Taking the first derivative of equation (33) the following expression for $f^{\prime}(t)$ is obtained

$$
\begin{aligned}
f^{\prime}(t)= & f_{1}^{\prime}(t)\left[1-H\left(t-t_{1}\right)\right]-f_{1}(t) \delta\left(t-t_{1}\right)+\cdots \\
& f_{m+1}^{\prime}(t) H\left(t-t_{m}\right)+f_{m+1}(t) \delta\left(t-t_{m}\right)+\cdots \\
& \sum_{i=1}^{m-1}\left\{f_{i+1}^{\prime}(t)\left[H\left(t-t_{i}\right)-H\left(t-t_{i+1}\right)\right]\right\}+\cdots \\
& \sum_{i=1}^{m-1}\left\{f_{i+1}(t)\left[\delta\left(t-t_{i}\right)-\delta\left(t-t_{i+1}\right)\right]\right\}
\end{aligned}
$$

where $\delta(t)$ in equation (35) is the Dirac delta function, which can be defined by

$$
\int_{a}^{b} h(t) \delta\left(t-t_{0}\right) \mathrm{d} t= \begin{cases}h\left(t_{0}\right) & \text { if } a<t_{0}<b, \\ 0 & \text { if } t_{0}<a \text { or } t_{0}>b\end{cases}
$$

Also in equation (35) we have considered the property $H^{\prime}(t)=\delta(t)$. That is to say, the distributional derivative of the Heaviside step function is the Dirac delta function (Quarteroni \& Valli, 2008).

In the case where the loading profile is piecewise smooth, computation of functions $p_{n}(t)$ defined by equation (32) would require substitution of equation (35) within equation (32).

Once the fluid pressure along the saturated column is computed by equation (27), the vertical displacement $u(z, t)$ can be derived by means of equation (19) as

$$
\begin{aligned}
u(z, t)= & \frac{f(t)(L-z)}{\lambda+2 \mu}-\cdots \\
& \quad \frac{1}{\lambda+2 \mu} \sum_{n=0}^{\infty} \frac{2 L}{(1+2 n) \pi} p_{n}(t) \cos \left[\frac{(1+2 n) \pi z}{2 L}\right]
\end{aligned}
$$

where $p_{n}(t)$ are again defined by equation (32).
Summarising, in order to compute the pore pressure distribution $p(z, t)$ and the vertical displacement $u(z, t)$ within a saturated poroelastic column subjected to a general timedependent loading $f(t)$, the steps described below should be followed.

Step 1. Define column length, Lamé constants (or oedometer modulus), Darcy permeability, acceleration of gravity and intrinsic density of water. Define also the external loading profile $f(t)$.

Step 2. Compute the following definite integral

$$
\int_{0}^{t} \mathrm{e}^{-\aleph_{n}(t-\tau)} f^{\prime}(\tau) \mathrm{d} \tau
$$

If the loading profile $f(t)$ is smooth this computation can be done directly. If the loading profile $f(t)$ is piecewise smooth, it is advised to rewrite this profile by means of the Heaviside function, as in equation (33), before computing the integral.

Step 3. Compute $p_{n}(t)$ by equation (32).

Step 4. Add up a finite number of terms in equations (27) and (37) to obtain the pore pressure distribution and the vertical displacement along the column, respectively.

The solution strategy, which is defined by steps 1 to 4 above, might be considered for any loading profile $f(t)$, whether the external load is smooth or piecewise smooth. The integral in step 2 can be performed by parts in most of the practical cases. If the external loading $f(t)$ is too unusual, this integral might be computed numerically by means of a standard quadrature rule. The number of terms to be considered in equations (27) and (37) should be established in such a way that further inclusion of more terms produces no changes in the solution.

\section{COMPARISON WITH ANALYTICAL SOLUTIONS}

In this section, the proposed solution strategy is applied to four different loading profiles: constant, single ramp, cyclic square and cyclic haversine. In order to assess the accuracy and robustness of the proposed strategy, the results are compared with the solutions derived by other researchers. All results are plotted as dimensionless.

The first case studied refers to the classical theory developed by Terzaghi (1925) for one-dimensional consolidation. The solution proposed by Terzaghi $(1925,1943)$ was obtained under constant loading. Therefore, in the proposed solution, it is necessary to consider $f^{\prime}(t)=0$ in equation (32) for the whole consolidation process. In this way, Terzaghi's solution is reproduced under the scope of the proposed strategy when coefficients $p_{n}(t)$ are

$$
p_{n}(t)=\frac{4 \mathrm{e}^{-\aleph_{n} t} f(0)}{\pi(2 n+1)}
$$

where $\aleph_{n}=c_{\mathrm{V}}[(1+2 n) \pi / 2 L]^{2}$ while $c_{\mathrm{v}}=k(\lambda+2 \mu) / \rho g$ is the coefficient of consolidation in the vertical direction. The value of $f(0)$ in equation (38) corresponds to the initial value of the load profile, which is held constant in the present case of analysis.

Substituting equation (38) into equation (27) provides the analytical solution for the pore fluid pressure distribution $p(z, t)$. Substituting equation (38) into equation (37) provides the vertical displacement $u(z, t)$.

Comparison between the solution proposed in the present work and the analytical solution proposed by Terzaghi (1943) is shown in Fig. 3.

The curves in Fig. 3, related to the proposed solution, were obtained with equation (27) considering only the first five terms. 


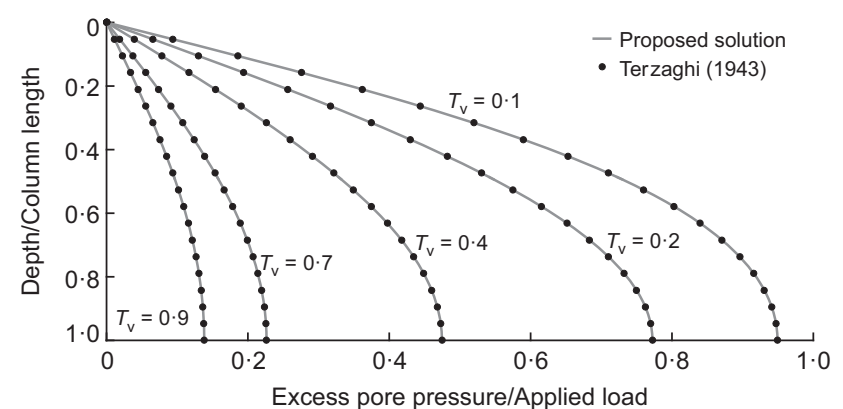

Fig. 3. Comparison of the proposed solution with Terzaghi (1943). Isochrones for different values of time factor $T_{v}=\left(c_{v} t\right) / L^{2}$

The second case studied refers to a ramp loading followed by a constant value of applied stress. In other words, the applied load at the top of the column increases linearly from zero up to a value $q$ attained at time $t=t_{\mathrm{c}}$ and then remains constant in time. This case was initially studied by Olson (1977), who proposed some analytical expressions to describe the phenomenon.

This load profile is piecewise smooth. Following equation (33) this load can be written as follows

$$
f(t)=\frac{q t}{t_{\mathrm{c}}}\left[1-H\left(t-t_{\mathrm{c}}\right)\right]+q H\left(t-t_{\mathrm{c}}\right)
$$

Taking the first derivative of equation (39), the following expression for $f^{\prime}(t)$ is obtained

$$
f^{\prime}(t)=\frac{q}{t_{\mathrm{c}}}\left[1-H\left(t-t_{\mathrm{c}}\right)\right]-\frac{q t}{t_{\mathrm{c}}} \delta\left(t-t_{\mathrm{c}}\right)+q \delta\left(t-t_{\mathrm{c}}\right)
$$

where $H(t)$ is the Heaviside function defined by equation (34), while $\delta(t)$ is the Dirac delta function defined by equation (36). Substituting equation (40) into equation (32), bearing in mind that $f(0)=0$ in this second case studied, the following expression is obtained for the coefficients $p_{n}(t)$

$$
p_{n}(t)=\frac{4 q h(t)}{\pi(2 n+1) t_{\mathrm{c}} \aleph_{n}}
$$

with

$$
h(t)= \begin{cases}\left(1-\mathrm{e}^{-\aleph_{n} t}\right) & \text { if } t<t_{\mathrm{c}} \\ \left(\mathrm{e}^{-\aleph_{n}\left(t-t_{\mathrm{c}}\right)}-\mathrm{e}^{-\aleph_{n} t}\right) & \text { if } t \geq t_{\mathrm{c}}\end{cases}
$$

where $\aleph_{n}=c_{\mathrm{V}}[(1+2 n) \pi / 2 L]^{2}$, while $\quad c_{\mathrm{v}}=k(\lambda+2 \mu) / \rho \boldsymbol{g}$ is the coefficient of consolidation in the vertical direction. Substituting equation (41) into equation (27) provides the analytical solution for the pore fluid pressure distribution $p(z, t)$. Substituting equation (41) into equation (37) provides the vertical displacement $u(z, t)$.

The comparison between the present solution and the analytical solution proposed by Olson in 1977, for a ramp loading profile with $t_{\mathrm{c}}=0 \cdot 2\left(L^{2} / c_{\mathrm{v}}\right)$, is shown in Fig. 4 .

In this second case studied, five terms were also considered in equation (27) to compute the pore pressure distribution along the column.

Taking into account the way in which the present approach has been applied to the constant and ramp loadings, it is clear that a non-periodic loading profile does not need to be converted into a periodic one, as required in the paper by Conte \& Troncone (2006). On comparing further with the solution proposed by Conte \& Troncone (2006), it is found that in the proposed approach there is no need to represent

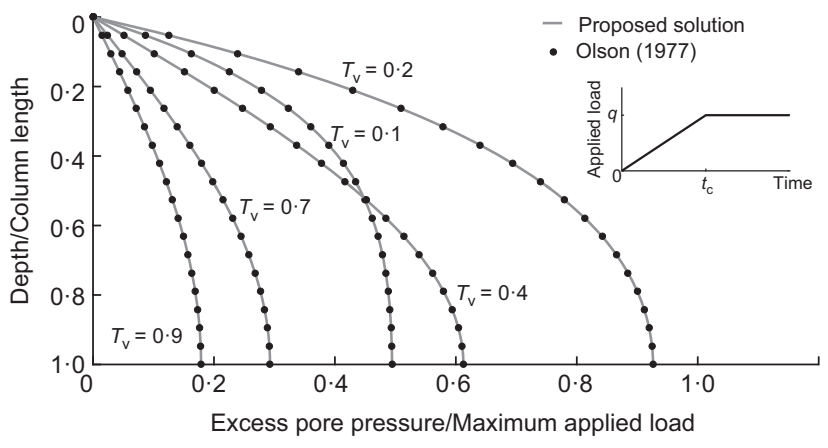

Fig. 4. Comparison of the proposed solution with Olson (1977). Isochrones for different values of time factor $T_{\mathrm{v}}=\left(c_{\mathrm{v}} t\right) / L^{2}$. Ramp loading profile with $t_{\mathrm{c}}=0 \cdot 2\left(L^{2} / c_{\mathrm{v}}\right)$

the loading profile as a classical Fourier series, whatever form it takes. However, classical Fourier representation is required in Conte and Troncone's solution. In addition, there is no need to start from zero pore pressure; therefore, a time $t_{a}$ used in Conte \& Troncone (2006), to account for the initial condition, is not required in the present approach. It is also observed that no time $t_{b}$ needs to be defined in order to account for a final time where the consolidation process is expected to be developed in practice. Under the scope of these comments, the proposed solution in this work does not require extra parameters $\left\{t_{a}, t_{b}\right\}$ to define the loading profile, and seems to be easier and more general than the one obtained by Conte and Troncone in 2006.

In the third case studied, a cyclic square loading profile is analysed. This case has been studied by many researchers. Among them, Baligh \& Levadoux (1978) and Conte \& Troncone (2006), proposed some analytical expressions to describe the response under this type of cyclic loading.

A cyclic square loading of period $t_{\mathrm{c}}, s+1$ complete cycles and amplitude $q$, can be written as

$$
f(t)= \begin{cases}q & \text { if } \quad i t_{\mathrm{c}}<t \leq i t_{\mathrm{c}}+\frac{t_{\mathrm{c}}}{2} \\ 0 & \text { if } \quad i t_{\mathrm{c}}+\frac{t_{\mathrm{c}}}{2}<t<(i+1) t_{\mathrm{c}}\end{cases}
$$

for $i \in\{0, \ldots, s\}$. As in the second case studied, the cyclic square loading defined by equation (42) is piecewise smooth. Therefore, following equation (33), this load can be written as follows

$$
\begin{aligned}
f(t)= & q\left[1-H\left(t-\frac{t_{\mathrm{c}}}{2}\right)\right]+\cdots \\
& \sum_{i=1}^{s} q\left\{H\left(t-i t_{\mathrm{c}}\right)-H\left[t-\left(i t_{\mathrm{c}}+\frac{t_{\mathrm{c}}}{2}\right)\right]\right\}
\end{aligned}
$$

Taking the first derivative of equation (43), the following expression for $f^{\prime}(t)$ is obtained

$$
\begin{aligned}
f^{\prime}(t)= & -q \delta\left(t-\frac{t_{\mathrm{c}}}{2}\right)+\cdots \\
& \sum_{i=1}^{s} q\left\{\delta\left(t-i t_{\mathrm{c}}\right)-\delta\left[t-\left(i t_{\mathrm{c}}+\frac{t_{\mathrm{c}}}{2}\right)\right]\right\}
\end{aligned}
$$

where $\delta(t)$ is the Dirac delta function defined by equation (36). Taking into account that $f(0)=q$ in this third case studied, substituting equation (44) into equation (32), the following expression is obtained for the coefficients $p_{n}(t)$

$$
p_{n}(t)=\frac{4 q}{\pi(2 n+1)}\left[\mathrm{e}^{-\aleph_{n} t}+h(t)\right]
$$


where

$$
h(t)=\left\{\begin{array}{l}
\sum_{i=0}^{m-1}\left[\mathrm{e}^{-\aleph_{n}\left[t-(i+1) t_{\mathrm{c}}\right]}-\mathrm{e}^{-\aleph_{n}\left\{t-\left[i t_{\mathrm{c}}+\left(t_{\mathrm{c}} / 2\right)\right]\right\}}\right] \\
\quad \text { if } m t_{\mathrm{c}}<t \leq \frac{(2 m+1) t_{\mathrm{c}}}{2} \\
\sum_{i=0}^{m-1}\left[\mathrm{e}^{-\aleph_{n}\left[t-(i+1) t_{\mathrm{c}}\right]}-\mathrm{e}^{-\aleph_{n}\left\{t-\left[t_{\mathrm{c}}+\left(t_{\mathrm{c}} / 2\right)\right]\right\}}\right]-\cdots \\
\mathrm{e}^{-\aleph_{n}\left\{t-\left[m t_{\mathrm{c}}+\left(t_{\mathrm{c}} / 2\right)\right]\right\}} \quad \text { if } \frac{(2 m+1) t_{\mathrm{c}}}{2}<t<(m+1) t_{\mathrm{c}}
\end{array}\right.
$$

for a fixed $m \in\{0, \ldots, \quad s\}$. In equation (45), $\aleph_{n}=c_{\mathrm{V}}[(1+2 n) \pi / 2 L]^{2}$, while $c_{\mathrm{v}}=k(\lambda+2 \mu) / \rho g$ is the coefficient of consolidation in the vertical direction. Again, substituting equation (45) into equation (27) provides the analytical solution for the pore fluid pressure distribution $p(z, t)$. Substituting equation (45) into equation (37) provides the vertical displacement $u(z, t)$.

Comparison between the solution derived in the present work and the analytical solution proposed by Balig and Levadoux in 1978, for the case of a cyclic square loading with period $t_{\mathrm{c}}=0 \cdot 1\left(L^{2} / c_{\mathrm{v}}\right)$, is shown in Fig. 5 .

Once more, in this third case studied, five terms were also considered in equation (27) to compute the pore pressure distribution along the column.

In the fourth and final case studied, a cyclic haversine loading is analysed. This case was studied by Razouki et al. (2013), proposing an analytical expression to describe the response under this type of cyclic loading.

Constant, single ramp and cyclic square loadings are quite common in engineering practice. However, this is not the case for haversine loadings. Nevertheless, Barskdale (1971) and Huang (1993) reported that it is reasonable to assume the stress pulse at a given point in the pavement of highways and airports to be a haversine loading, whose amplitude $q$ and period $t_{\mathrm{c}}$ depends on the vehicle speed and the depth of the point below the pavement surface. Following Huang (1993), the profile $f(t)$ for a haversine loading might be given as

$$
f(t)=q \sin ^{2}\left(\frac{\pi t}{t_{\mathrm{c}}}\right)
$$

This type of loading profile is smooth. Therefore, direct application of equation (32) can be considered. Taking the time derivative of equation $(46), f^{\prime}(t)$ has the following expression for a haversine loading

$$
f^{\prime}(t)=\frac{q \pi}{t_{\mathrm{c}}} \sin \left(\frac{2 \pi t}{t_{\mathrm{c}}}\right)
$$

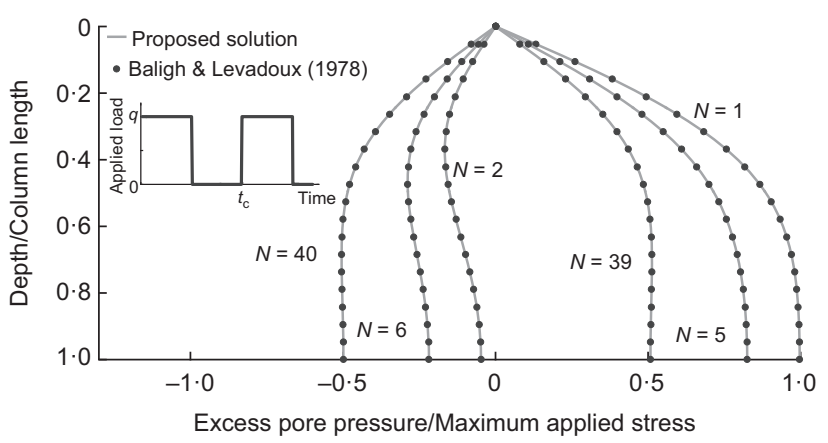

Fig. 5. Comparison of the proposed solution with Baligh \& Levadoux (1978). Isochrones for different values of half cycles $N$. Cyclic square loading with period $t_{\mathrm{c}}=0 \cdot 1\left(L^{2} / c_{\mathrm{v}}\right)$
Bearing in mind that $f(0)=0$ in this fourth case studied, substituting equation (47) into equation (32) and computing the integral therein, the following expression is obtained for the coefficients $p_{n}(t)$

$$
p_{n}(t)=\gamma\left[\sin \left(\frac{2 \pi t}{t_{\mathrm{c}}}\right)-\frac{2 \pi}{\aleph_{n} t_{\mathrm{c}}} \cos \left(\frac{2 \pi t}{t_{\mathrm{c}}}\right)+\frac{2 \pi}{\aleph_{n} t_{\mathrm{c}}} \mathrm{e}^{-\aleph_{n} t}\right]
$$

where $\quad \gamma=4 q \aleph_{n} t_{\mathrm{c}} /\left[(2 n+1)\left(\aleph_{n}^{2} t_{\mathrm{c}}^{2}+4 \pi^{2}\right)\right], \quad \aleph_{n}=c_{\mathrm{v}}[(1+2 n)$ $\pi / 2 L]^{2}$ while $c_{\mathrm{v}}=k(\lambda+2 \mu) / \rho g$ is the coefficient of consolidation in the vertical direction. Substituting equation (48) into equation (27) provides the analytical solution for the pore fluid pressure distribution $p(z, t)$. Substituting equation (48) into equation (37) provides the vertical displacement $u(z, t)$.

Comparison between the solution derived in the present work and the one proposed by Razouki and coworkers (Razouki et al., 2013), for the case of a cyclic haversine loading with period $t_{\mathrm{c}}=0 \cdot 1\left(L^{2} / c_{\mathrm{v}}\right)$, is shown in Fig. 6 .

Once more, in this fourth case studied, five terms were considered in equation (27) to compute the pore pressure distribution along the column.

In Figs 3-6 no differences have been observed between the semi-analytical solution proposed in the present work and those presented by other researchers (Terzaghi, 1943; Olson, 1977; Baligh \& Levadoux, 1978; Conte \& Troncone, 2006; Razouki et al., 2013) for the different loading profiles: constant, single ramp, cyclic square and cyclic haversine.

Regarding the number of terms to be considered in equations (27) and (37), the following remark should be taken into account. Pore pressure at the top of the column is zero over the whole consolidation process. However, if a steep loading is applied at this upper boundary and a soil with a low consolidation coefficient is considered, then the pore pressure near the perfectly drained boundary may develop an instantaneous steep gradient. The accuracy of the response depends strongly on this gradient. The steeper the pore pressure gradient is, the greater the number of terms that need to be considered in equations (27) and (37).

\section{CONCLUSIONS}

A (four-step) strategy to obtain an analytical solution to the well-known consolidation equation in a one-dimensional saturated poroelastic medium, under a general loading profile, has been developed in the present work. Once a specific external loading $f(t)$ is considered, whether this external load is smooth or piecewise smooth, the proposed strategy requires the following steps.

Step 1. Define the column length, Lamé constants (or oedometer modulus), Darcy permeability, acceleration of

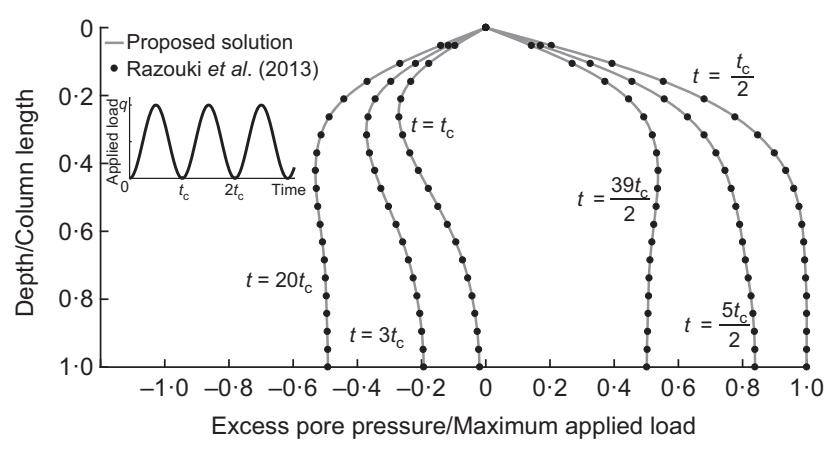

Fig. 6. Comparison of the proposed solution with Razouki et al. (2013). Isochrones for different values of time $t$. Cyclic haversine loading with period $t_{\mathrm{c}}=0 \cdot 1\left(L^{2} / c_{\mathrm{v}}\right)$ 
gravity and intrinsic density of water. Define also the external loading profile $f(t)$.

Step 2. Compute the following definite integral

$$
\int_{0}^{t} \mathrm{e}^{-\aleph_{n}(t-\tau)} f^{\prime}(\tau) \mathrm{d} \tau
$$

If the loading profile $f(t)$ is smooth, this computation can be done directly. If the loading profile $f(t)$ is piecewise smooth, it is advised to rewrite this profile by means of the Heaviside function equation (33) before computing the integral.

Step 3. Compute $p_{n}(t)$ by equation (32).

Step 4. Add up a finite number of terms in equation (27) and equation (37) to obtain the pore pressure distribution and the vertical displacement along the column, respectively.

The proposed four-step strategy might be considered to obtain the solution for the one-dimensional consolidation equation under any loading profile $f(t)$, whether the external load is smooth or piecewise smooth.

Comparing the proposed solution with other approaches, it is found that any non-periodic loading profile does not need to be converted into a periodic one in the present work. In addition, there is no need, in the proposed approach, to represent the loading profiles as a classical Fourier series. In the same way, there is no need to start from zero pore pressure. Therefore, a special time instant to account for the initial condition is not required in the present approach. It is also observed that no time instant is needed to account for a final time where the consolidation process is expected to be developed in practice. Within the scope of these comments, the proposed solution in this work does not require extra parameters to define the loading profile, is straightforward and can be applied to truly general time-dependent loading profiles.

Armed with the present strategy, there is no need to perform the complete eigenfunction expansion method for new loading profiles. Steps 1 to 4 above are all that is required to obtain the solution for the one-dimensional consolidation equation under any loading profile. In this sense, the solution procedure simplifies in terms of practical geotechnical engineering. Comparison with the analytical solution previously obtained by other researchers is highly satisfactory.

\section{ACKNOWLEDGEMENTS}

The authors gratefully acknowledge the support from the Spanish Ministry of Economy, Industry and Competitiveness with the reference BIA2016-76253-P (ALAS). In addition, the first author would like to thank the Jose Entrecanales Ibarra Foundation for the mobility grant funding his visiting scholar stay at Stanford University.

\section{NOTATION}

$c_{\mathrm{v}}$ coefficient of consolidation in vertical direction

$\mathrm{d} v$ representative element volume of the mixture

$\mathrm{d} v^{\alpha}$ element volume of the phase $\alpha$

$f(t)$ time-dependent loading

$f_{n}(t) \quad$ Fourier coefficients

$g$ acceleration of gravity

$H(t)$ Heaviside function

I second-order identity tensor

$k^{f}, k \quad$ Darcy permeability

$L$ column length

$N$ number of half square cycles

$n$ outward unit normal vector

$n$ non-negative integer

$n^{\alpha}(\boldsymbol{x}, t) \quad$ volume fraction of phase $\alpha$
$P^{\alpha} \quad$ particles of phase $\alpha(\alpha=f$ fluid phase, $\alpha=s$ skeleton phase)

$p^{f}, p \quad$ pore fluid pressure

$\hat{p}^{f}, \hat{q}$ prescribed pore fluid pressure and flux

$q$ maximum applied load

$T_{\mathrm{v}}$ time factor

$t$ time

$t_{a}, t_{b} \quad$ parameters required in Conte \& Troncone (2006)

$t_{\mathrm{c}}$ time at which maximum load $q$ is applied in ramp load, period of cyclic loading

$t_{i} \quad$ simple jump discontinuity

$u$ vertical displacement

$\boldsymbol{u}^{s} \quad$ skeleton displacement vector

$\hat{\boldsymbol{u}}^{s}, \hat{\boldsymbol{t}}$ prescribed skeleton displacement and total stresses

$v^{\alpha}$ velocity of the particles $P^{\alpha}$

$\boldsymbol{X}^{\alpha}$ position in the reference configuration of particle $P^{\alpha}$ at time $t_{0}$

$\boldsymbol{x}$ position in the current configuration

$y_{n}$ eigenfunctions of the Sturm-Liouville problem

$z$ vertical direction

$\delta(t) \quad$ Dirac delta function

$\partial \Omega \quad$ boundary of the domain $\Omega$

$\partial \Omega_{p^{f}}$ part of the boundary $\Omega$ with prescribed pore fluid pressure

$\partial \Omega_{q} \quad$ part of the boundary $\Omega$ with prescribed pore fluid flux

$\partial \Omega_{t} \quad$ part of the boundary $\Omega$ with prescribed total stresses

$\partial \Omega_{\boldsymbol{u}^{s}} \quad$ part of the boundary $\Omega$ with prescribed skeleton displacement

$\boldsymbol{\varepsilon} \quad$ small strain tensor

$\rho^{f R}, \rho$ pore fluid intrinsic density

$\boldsymbol{\sigma}$ total Cauchy stress tensor

$\sigma$ total vertical stress

$\boldsymbol{\sigma}^{\prime}$ effective Cauchy stress tensor

$\lambda, \mu$ macroscopic Lamé constants

$\lambda_{n}$ eigenvalues of the Sturm-Liouville problem

$\chi^{s}, \chi^{f}$ motion of the skeleton and fluid

$\Omega$ general domain

\section{REFERENCES}

Baligh, M. M. \& Levadoux, J. N. (1978). Consolidation theory for cyclic loading. J. Geotech. Engng Div. ASCE 104, No. 4, 415-431.

Barskdale, R. G. (1971). Compressive stress pulse times in flexible pavements for use in dynamic testing. Highway Res. Rec. $\mathbf{3 4 5}$, No. 4, 32-44

Biot, M. A. (1941). General theory of three-dimensional consolidation. J. Appl. Physics 12, No. 2, 155-164.

Conte, E. \& Troncone, A. (2006). One-dimensional consolidation under general time-dependent loading. Can. Geotech. J. 43, No. 11, 1107-1116.

de Boer, R. (2005). Trends in continuum mechanics of porous media. Berlin/Heidelberg, Germany: Springer-Verlag.

de Boer, R., Ehlers, W. \& Zhangfang, L. (1993). One-dimensional transient wave propagation in fluid-saturated incompressible porous media. Arch. Appl. Mech. 63, No. 1, 59-72.

Farlow, S. J. (1982). Partial differential equations for scientist and engineers. New York, NY, USA: Dover.

Favaretti, M. \& Soranzo, M. (1995). A simplified consolidation theory in cyclic loading condition. In Compression and consolidation of clayey soils: proceedings of the international symposium on compression and consolidation of clayey soils, IS-Hiroshima '95 (eds H. Yoshikuni and O. Kusakabe), pp. 405-409. Rotterdam, the Netherlands: Balkema.

Geng, X., Xu, C. \& Cai, Y. (2006). Non-linear consolidation analysis of soil with variable compresibility and permeability under cyclic loadings. Int. J. Numer. Analyt. Methods Geomech. 30, No. 8, 803-821.

Guan, S.-H., Xie, K.-H. \& Hu, A.-F. (2003). Analysis of onedimensional consolidation behavior of soils under lowfrequency cyclic loading. Rock Soil Mech. 24, No. 5, 849-853.

Huang, Y. H. (1993). Pavement analysis and design. Englewwod Cliffs, NJ, USA: Prentice-Hall.

Hughes, T. J. R. (2000). The finite element method. New York, NY, USA: Dover. 
Logan, J. D. (2004). Applied partial differential equations. New York, NY, USA: Springer-Verlag.

Olson, R. E. (1977). Consolidation under time-dependent loading. J. Geotech. Engng Div. ASCE 103, No. 1, 55-60.

Quarteroni, A. \& Valli, A. (2008). Numerical approximation of partial differential equations. Berlin/Heidelberg, Germany: Springer-Verlag.

Razouki, S. S. \& Schanz, T. (2011). One-dimensional consolidation under haversine repeated loading with rest period. Acta Geotechnica 6, No. 1, 13-20.

Razouki, S. S., Bonnier, P., Datcheva, M. \& Schanz, T. (2013). Analytical solution for $1 \mathrm{D}$ consolidation under haversine cyclic loading. Int. J. Numer. Analyt. Methods Geomech. 37, No. 14, 2367-2372.

Selvadurai, A. P. S. (2000). Partial differential equations in mechanics I. Berlin/Heidelberg, Germany: Springer-Verlag.
Stickle, M. M., Yague, A. \& Pastor, M. (2016). Free finite element approach for saturated porous media: consolidation. Mathematical Problems Engng 2016, article ID 4256079.

Taylor, D. W. (1948). Fundamentals of soil mechanics. New York, NY, USA: John Wiley Sons.

Terzaghi, K. (1925). Erdbaumechanik. Vienna, Austria: F. Deuticke (in German).

Terzaghi, K. (1943). Theoretical soil mechanics. New York, NY, USA: John Wiley Sons.

Wood, D. M. (2004). Geotechnical modelling. London, UK and New York, NY, USA: Spon Press, Taylor \& Francis Group.

Zienkiewicz, O. C., Chang, C. T. \& Bettes, P. (1980). Drained, undrained, consolidation and dynamic behaviour assumptions in soils. Géotechnique 30, No. 4, 385-395, https://doi.org/ 10.1680/geot.1980.30.4.385. 